\title{
Animal research in short
}

AnimalResearch.Info is a "global resource for scientific evidence in animal research" formed by "an international collaboration of scientists and researchers" with the stated goal "to build an understanding about how and why animals are used in science and medicine."

The site begins with information on hepatitis C, HIV and AIDS and the use of nonhuman primates and dogs in research and includes a timeline of medical developments. The most relevant sections for animal researchers are those on 'Designing Research,' 'Medical Advances' and 'Drug Development.' Explanatory text is presented in simple paragraphs, and key information is organized into lists and can be searched by keyword and filtered by variables, including scientist name, animal, research field, country or medical application. The 'Designing Research' section is subdivided into six subtopics: 'Why Animals are Used,' 'Research Animals,' 'Experimental Design \& Analysis,' 'Chemical Toxicology,' 'Alternatives and the 3Rs' and 'Misconceptions.' 'Research Animals' presents information on animal models organized in an alphabetized list from 'C. elegans (nematode worm)' down to 'zebrafish.'

The 'Medical Advances' section is subdivided into five subtopics. First is the 'Timeline,' which begins in 1881 with the 'Germ Theory of Disease' and Louis Pasteur and ends in 2008 with the 'First tissue-engineered whole-organ transplant', transplantation of an autologous stem cell engineered trachea into a human. Alphabetized topics range from 'Artificial heart valves' to 'Vitamin A discovered.' The second 'Medical Advances' subtopic is 'Diseases \& Research,' which spans 'AIDS \& HIV' research in mice or primates to 'Whooping cough (pertussis) vaccine' in mice and rabbits. Next in 'Medical Advances' are the Nobel Prizes pages, which include the 2013 prizes for Physiology or Medicine and date back to the 1901 prize to Emil von Behring for discovering that animals immunized with diphtheria toxin produced an anti-toxin that could be used as a vaccine against the infectious agent. The 'Veterinary Medicine' pages cover fewer topics, beginning with 'Bovine tuberculosis vaccine' and ending with 'Vaccine development.'

The 'Drug Development' tab includes three subtopics: 'The Process,' 'Safety \& Testing' and 'Modernising the Process.' 'The Process' briefly explains drug development from computer models through to 'Clinical Trials' and licensure of medications. 'Safety \& Testing' includes information on toxicity testing and ends on a paragraph about 'alternative methods.'

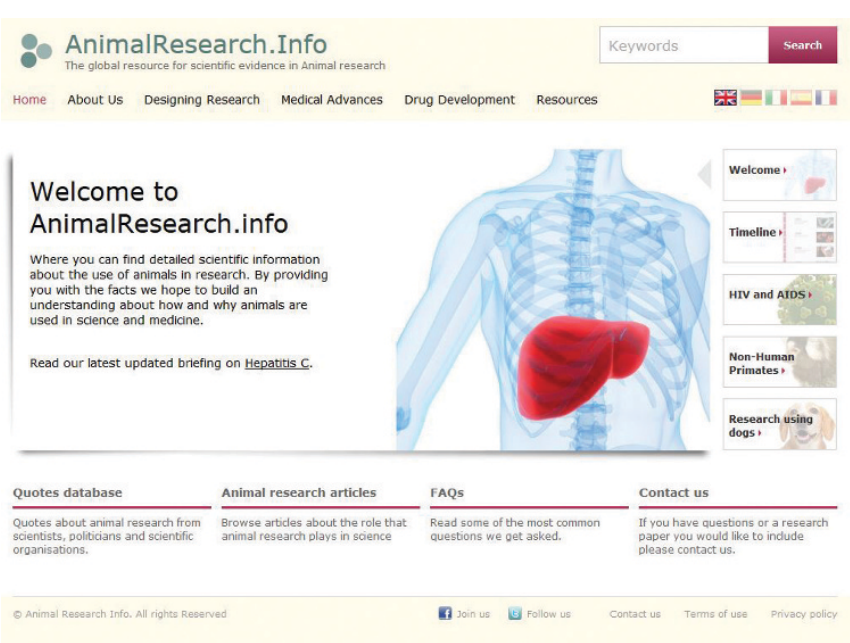

The 'Resources' page begins with a subpage on 'FAQs' answering common questions about animal research. Next is a searchable database of quotes either supportive of or opposed to animal research. These are very useful for popping into a paper or lecture. There are four book reviews on the 'Book Reviews' page, two biographies on the 'Biographies' page, a long list of 'Research Organisations' (most located in the UK), samples of animal welfare legislation under 'Regulation \& Legislation' and an extensive list of 'Links.'

The 'Contribute' page, located under the 'About Us' tab, is not a solicitation for monetary contributions but rather a solicitation for articles for the website or additions to current information on the site.

This is a great site where anyone who doubts the validity of animal research can learn why and how animals are used in research and find out about medical advances that have occurred as a result of animal research. It also provides a good history of animal research. AnimalResearch.info is available in English, German, Italian, Spanish and French.

Although this site is useful and informative, particularly for the public, many of the articles do not contain up-to-date references (this reviewer's one complaint). A website spokesperson assured Lab Animal that the site is being updated.

\section{http://www.animalresearch.info/}
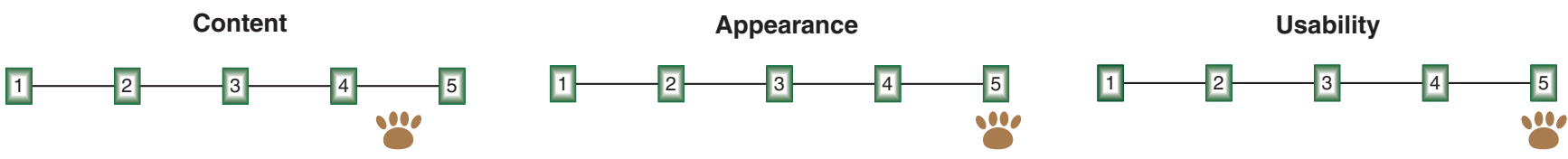